\title{
Multi-omics analysis reveals cross-organism interactions in coral holobiont
}

Toru Maruyama ${ }^{1,2}$, Michihiro Ito ${ }^{3}$, Satoshi Wakaoji ${ }^{1}$, Yusuke Okubo ${ }^{1}$, Keigo Ide ${ }^{1,2}$, Yohei Nishikawa ${ }^{4}$, Hiroyuki Fujimura ${ }^{5}$, Shoichiro Suda ${ }^{5}$, Yoshikatsu Nakano ${ }^{6,7}$, Noriyuki Satoh ${ }^{8}$, Chuya Shinzato ${ }^{9}$, Kei Yura ${ }^{1,2,10}$, Haruko Takeyama ${ }^{1,2,4,11^{*}}$

${ }^{1}$ Department of Life Science \& Medical Bioscience, Graduate School of Advanced Science \& Engineering, Waseda University, Shinjuku, Tokyo, Japan

${ }^{2}$ Computational Bio-Big Data Open Innovation Lab., National Institute of Advanced Science and Technology, Shinjuku, Tokyo, Japan

${ }^{3}$ Center of Molecular Biosciences, Tropical Biosphere Research Center, University of the Ryukyus, Nishihara, Okinawa, Japan

${ }^{4}$ Research Organization for Nan and Life Innovation, Waseda University, Shinjuku, Tokyo, Japan

${ }^{5}$ Department of Chemistry, Biology and Marine Science, University of the Ryukyus, Nishihara, Okinawa, Japan

${ }^{6}$ Tropical Biosphere Research Center, Sesoko Station, University of the Ryukyus, Motobu, Okinawa, Japan

${ }^{7}$ Marine Science Section, Okinawa Institute of Science and Technology Graduate University, Onna, Okinawa, Japan

${ }^{8}$ Marine Genomics Unit, Okinawa Institute of Science and Technology Graduate University, Onna, Okinawa, Japan

${ }^{9}$ Atmosphere and Ocean Research Institute, The University of Tokyo, Chiba, Japan

${ }^{10}$ Graduate School of Humanities and Sciences, Ochanomizu University, Bunkyo, Tokyo, Japan 
${ }^{11}$ Institute for Advanced Research of Biosystem Dynamics, Waseda Research Institute for Science and Engineering, Shinjuku, Tokyo, Japan

*Correspondence: haruko.takeyma@waseda.jp

\begin{abstract}
Corals create an ecosystem, called a holobiont, with intracellular algae (zooxanthellae) and resident bacteria. Zooxanthellae and some bacteria play major roles in the physiological properties of the coral host. However, because of the difficulties in experimental verification of cross-organism interactions, the mechanisms underpinning these interactions are largely unknown. To address this, we here generated and then analyzed multi-omics datasets for corals, zooxanthellae, and bacteria collected at Okinawa, Japan, from November 2014 to September 2016. Using cross-organism co-expression analysis, we successfully characterized the host-alga relationship in the coral holobiont. Specifically, we observed that the coral host dominates the zooxanthellae. The multi-omics analysis also suggested that infection with coral-associated bacteria Endozoicomonas likely involves coral-like ephrin ligands, triggering an immune response of the coral host. This study highlights the potential of the multi-omics approach to elucidate coral-microbe interactions.
\end{abstract}

\title{
Keywords
}

Symbiosis, Multi-omics, Coral holobiont, Endozoicomonas 


\section{Introduction}

Corals form an ecosystem, called a holobiont, with intracellular dinoflagellates and resident microbiota. The intracellular dinoflagellates, zooxanthellae, play critical roles in the coral host, as they are important photosynthate providers. For instance, zooxanthellae produce more than $90 \%$ of metabolites required for coral respiration, via photosynthesis ${ }^{1,2}$. These dinoflagellates also synthesize other beneficial substances, such as mycosporine-like amino acids ${ }^{3,4}$. Collapse of the symbiotic relationship is detrimental to the coral and has been a major factor in the worldwide decline in coral populations ${ }^{5}$. The breakdown of symbiosis, known as coral bleaching, is triggered by stress factors such as rising sea temperature; however, the mechanism underlying this phenomenon is far from being elucidated ${ }^{6}$. Further, while the above symbiotic relationship is generally considered mutually beneficial ${ }^{1,2}$, some controversy surrounds this notion, and some researchers hypothesize that the coral host exerts a dominant control over the intracellular symbiont ${ }^{7}$.

A close relationship between the coral and bacteria is also expected, as first proposed in coral probiotic hypothesis ${ }^{8}$. It has been reported that the bacterial community structure changes in response to the conditions to which the coral host is subjected, including bleaching ${ }^{9}$, disease ${ }^{10-13}$, and temperature ${ }^{14}$. Further, alteration of the bacterial flora in coral mucus by antibiotics causes coral bleaching and death 15. The coral microbiome is completely different from that of the surrounding seawater ${ }^{16}$ and consists of commensal bacteria ${ }^{17,18}$. Bacteria from the Endozoicomonas genus have been identified in coral microbiomes worldwide and have been proposed to reside inside coral tissues and cells ${ }^{18,19}$. Further, it has been reported that the health of the coral host deteriorates when the Endozoicomonas population declines, suggesting that these microorganisms exert a beneficial effect 
on the coral host ${ }^{9,13,15}$. However, Endozoicomonas have been suggested to be pathogenic in fish ${ }^{20,21}$, and the mechanisms by which they contribute to coral health are largely unknown.

Although the relationship between the intracellular algae and resident bacteria is important for corals, the underlying molecular mechanisms have not been fully elucidated because of the difficulties associated with the experimental investigation of this complex biological system. Many intracellular algae and resident bacteria are unculturable $^{22,23}$, which hampers the attempts to set up an experimental model of symbiotic interactions. Consequently, an alternative, data-driven approach involving simultaneous omics profiling of the host and microbe, is used to understand such cross-organism interactions ${ }^{24}$. In coral research, simultaneous profiling of coral and zooxanthellae mRNA revealed a repertoire of genes in both organisms ${ }^{25}$. In the current study, we have extended the multi-omics approach to decipher interspecific crosstalk in the coral holobiont. We profiled the (i) coral and (ii) zooxanthellae transcriptome; (iii) 16S rRNA-based coral microbiome; and (iv) bacterial genome. Integrative analyses of the multi-omics datasets successfully highlighted complex interactions among corals, zooxanthellae and coral microbiome.

\section{Results}

\section{Multi-omics profiling of coral holobiont}

We collected the scleractinian coral Acropora tenuis at three sampling sites, Ishikawabaru (Is), Kyuusanbashi $(K y)$ and Sesoko-minami $(M i)$ around Sesoko Island, Okinawa, Japan (Supplementary Fig. 1), between November 2014 to September 2016. Is is located in a reef lagoon and is close to a sewage plant and a tuna farm. Mi and Ky are in open areas with low impact from human activity. We 
collected 63 samples from seven coral colonies (Supplementary Table 1). The corals showed signs of bleaching in August and September 2016 but appeared healthy at other sampling times (Supplementary Fig. 2).

We simultaneously generated multi-omics data of coral, zooxanthella, and bacterium for the collected samples (Fig. 1). Briefly, we extracted mRNAs of coral and zooxanthella from the total RNA of the holobiont and sequenced them simultaneously. We aligned the mRNA-seq sequence reads to the genome of $A$. tenuis $^{26}$ to determine the mRNA profiles of the coral hosts. For the dataset, 33.6$69.0 \%$ (average $52.6 \%$ ) of the reads were aligned to $A$. tenuis genome (Supplementary Table 2). We then extracted the unmapped sequence reads for de novo assembly to obtain zooxanthella transcripts. Finally, we analyzed the obtained 325,653 contigs by similarity searching against publicly available transcript sequences of corals and zooxanthellae ${ }^{4,26-29}$. Accordingly, 106,269 and 59,265 contigs shared similarity with the coral and zooxanthella sequences, respectively (Supplementary Fig. 3a), and were characterized by distinct GC content distributions (Supplementary Fig. 3b). The unclassified contigs were largely short (Supplementary Fig. 3c) and had a GC content close to that of corals (Supplementary Fig. 3b). Therefore, we concluded that the majority of the zooxanthella transcripts were successfully extracted using the described approach. We re-aligned the unmapped reads to the zooxanthella transcripts and determined the gene expression profiles of the intracellular algae. We also amplified the 16S rRNA V1-V2 region from the total holobiont RNA by RT-PCR and sequenced the resultant amplicons to obtain information on coral-associated bacterial communities. Finally, we analyzed the genome sequence of Endozoicomonas sp. ISHI1, isolated from $A$. tenuis at the Is site. 


\section{Season and colony-dependence of coral gene expression}

We analyzed the coral transcriptome using weighted gene co-expression network analysis (WGCNA) and identified 36 co-expression gene sets, henceforth referred to as "modules". Each module showed a different expression pattern, and their sizes ranged from 55 to 321 genes. Twenty modules showed seasonal variation, whereas the other 16 were differentially expressed in specific colonies (Fig. 2a,b). For example, the "green" module showed season-dependent expression and was highly expressed during summer (2015/08, 2016/08, and 2016/09) (Fig. 2a), while the "darkgrey" module was specifically expressed in Is3 colony regardless of the season (Fig. 2b).

Among the 20 season-dependent modules, 18 showed a significant correlation $(p<0.05)$ with at least one environmental parameter (Fig. $2 c)$. The expression of 15 modules was significantly correlated (or anti-correlated) with water temperature. Among them, the positively correlated modules were significantly associated with GPCR signaling (black, cyan, floralwhite, and green), organization of the extracellular matrix and bone (skyblue), ion channel activity (cyan), and the exosome/phagosome (brown4 and skyblue) (Supplementary Fig. 4a, and Supplementary Tables 3 and 4). The negatively correlated modules were significantly associated with protein/peptide degradation process (lightyellow, turquoise, and white), cell replication (blue and sienna3), and sphingolipid metabolism (orangered4) (Supplementary Fig. 4b, and Supplementary Tables 3 and 4). Two modules (skyblue3 and paleturquoise) were correlated with salinity and $\mathrm{pH}$; however, no significant functional enrichments were apparent. The "orange" module was significantly associated with spermatogenesis (Supplementary Table 3). Further, 
this module was upregulated in the spawning season (May 2015 and 2016), suggesting that it was indeed associated with the reproduction process.

Sixteen colony-dependent modules were not as well annotated as the season-dependent modules, as determined by similarity searches against Swiss?Prot and KEGG databases (Supplementary Fig. 5). Seven colony-dependent modules were significantly associated with the process of DNA integration, recombination, and transposition (royalblue, saddlebrown, darkred, lightcyan, steelblue, darkturquoise, and midnightblue) (Supplementary Fig. 6). These observations suggest that the modules involved in intraspecific variations were enriched with functionally unknown genes, with some of them acquired via genetic recombination and transposition.

\section{Seasonal changes in zooxanthella gene expression}

We also applied WGCNA to the zooxanthella transcriptome and identified 27 modules. Among them, 22 modules showed seasonal variation, whereas the remaining five were differentially expressed in specific colonies (Fig. 3a,b). To evaluate how changes in zooxanthella populations impacted the analysis, we examined the origin of zooxanthella clades that each module was derived from. More than $75 \%$ of all transcripts shared the highest similarity with the sequences of Clade C zooxanthellae (Cladocopium sp. clade $\mathrm{C}$ or Cladocopium goreaui) ${ }^{30}$, and every module exhibited a similar trend (Supplementary Fig. 7). This suggests that Cladocopium is the dominant zooxanthella type regardless of the season and colony and that the modules reflect transcriptional changes to a greater extent than zooxanthella population changes.

Among the 22 season-dependent modules, 18 were significantly correlated ( $p$ 
$<0.05$ ) with at least one environmental parameter (Fig. 3c). Eleven modules were significantly correlated (or anti-correlated) with water temperature, with eight modules positively correlated with water temperature. Of these eight modules, the "floralwhite" and "lightsteelblue1" modules were significantly associated with cilium movement; "plum2" and "skyblue" were significantly associated with photosynthesis; "skyblue3" was significantly associated with cell cycle; and "thistle2" was significantly associated with superoxide regulation (Supplementary Tables 5 and 6). In addition, the "brown4" module, which was negatively correlated with water temperature, was associated with the interleukin 17 signaling pathway (Supplementary Table 6). Finally, the "red" module was significantly associated with carbon fixation and nitrogen metabolism and was highly correlated with salinity (Supplementary Tables 5 and 6).

\section{Cross-species co-expression network analysis}

To determine the factors affecting the coral and zooxanthella transcriptomes, we calculated the correlations for their gene expression profiles. The gene expression profiles of the corals were similar among samples originating from the same colony (Supplementary Fig. 8a,d), while the profiles of zooxanthellae were similar among samples collected at the same sampling time (Supplementary Fig. 8b,e). These observations suggest that many genes are independently regulated in the coral hosts and in zooxanthellae.

We then investigated cross-species gene co-expression in the corals and zooxanthellae to estimate mechanisms underlying the coral-algal symbiosis. Specifically, to understand mechanisms of their interaction underpinning coral bleaching, we selected the modules that were significantly correlated with 
zooxanthella cell density (Supplementary Fig. 2) and calculated the co-expression among them (Fig. 4). Zooxanthella cell densities were positively correlated with coral modules associated with DNA replication (sienna3), cell cycle (blue), and sphingolipid biosynthesis (orangered4). The coral modules co-expressed with zooxanthella modules were associated with carbon fixation and nitrogen metabolism (red). However, the carbon fixation module (red) showed negative correlation with zooxanthella module associated with the DNA replication process (skyblue3). These findings suggest that carbon fixation by zooxanthellae contributes to the growth of the coral host, but it is not strongly linked to the growth of zooxanthellae themselves.

Zooxanthella cell densities were negatively correlated with coral modules associated with phagosomes (brown4), exosomes (byan), and zooxanthella modules associated with DNA replication (skyblue3), cilium movement (lightsteelbule1), and regulation of reactive oxygen species generation (thistle2). These observations suggest that zooxanthella cell density is actively regulated by the coral host via phagocytosis and exocytosis and that the expelled zooxanthellae may acquire motile behavior by retaining the flagella, as previously suggested ${ }^{2}$.

\section{Site and colony-dependent community structure of coral microbiome}

We analyzed the bacterial composition of coral microbiome and the surrounding seawater using total RNA-based 16S rRNA amplicon sequencing. The coral microbiome was mainly composed of microorganisms from the Rickettsiales family (Anaplasma-like) ${ }^{31}$, Endozoicomonas, and Cyanobacteria Group VIII (Fig. 5a). Further, it was dominated by 10 operational taxonomic units (OTUs) (Fig. 5b), which accounted for $>60 \%$ of the coral microbiome, with $<0.3 \%$ detected in the surrounding seawater. Principal coordinate analysis (PCoA) using unweighted UniFrac distance 
revealed that the data points for microbiomes from corals and seawater grouped separately (Fig. 5c).

Some genera and OTUs were specific to the sampling site or the colony. For instance, we only detected Cyanobacteria Group VIII at the Mi site (Fig. 5d). By contrast, we identified Anaplasma-like bacteria, which was reported as a marker of coral disease susceptibility ${ }^{32}$, at /s and Mi sites, except for the Is2 and Mi3 sites (Fig. 5e). Finally, we detected Endozoicomonas, a genus recognized as a commensal of diverse marine organisms, including corals at several geographic locations, mostly at the Is site, especially the Is1 and Is2 colonies (Fig. 5f). Distribution patterns of the species from the genus Endozoicomonas differed. We typically identified OTU3 and OTU5 at Is1 and Is2, whereas OTU13 and OTU549 were specific to Is2 (Fig. $5 \mathrm{~g}$ ). Further, the UniFrac distance analysis indicated the highest similarity among samples originating from the same colony or the same sampling site $(p<0.01$, PERMANOVA) (Supplementary Fig. 8c,f). In contrast with the gene expression profiles of corals and symbiotic algae, the composition of coral microbiome was strongly affected by the sampling site (Supplementary Fig. 8). This suggests that the microbiomes are regulated not only by the coral host but also by other factors that do not directly influence coral gene expression.

\section{Associations between coral microbiome and the coral host}

We only detected Anaplasma-like and Endozoicomonas bacteria in specific coral colonies (Fig. 5e,f). We therefore examined the genes differentially expressed in these colonies to determine the impact of each genus on the coral host. The analysis revealed that 1,500 and 1,425 genes were up or downregulated, respectively, in the colonies harboring Anaplasma-like bacteria (Is1, Is3, Mi1, and Mi2) (Supplementary 
Table 7). Further, 1,690 and 1,984 genes were up or downregulated in the colonies associated with Endozoicomonas (Is1 and Is2) (Supplementary Table 8). Gene sets related to immune reaction (e.g., positive regulation of interleukin 1 or regulation of killing of cells of other organisms) and vesicle formation (e.g., vesicles budding from membrane or phagolysosome assembly) were significantly enriched in the upregulated genes in colonies harboring Endozoicomonas (Supplementary Table 9). The upregulated genes included several P2X purinoceptor 7 (P2RX7) genes (Gene ID: MSTRG.12568 and MSTRG.9878) (Fig. 6a, Supplementary Table 9). P2RX7 is associated with host-pathogen interactions, such as elimination of intracellular parasites ${ }^{33}$, and is conserved in cnidarian species ${ }^{34}$. We speculated that Endozoicomonas triggers inflammatory reactions by activating host P2RX7. The upregulated genes also included ephrin receptor genes (Gene ID: MSTRG.5097 and MSTRG.31636) (Fig. 6b, Supplementary Table 8). Ephrin ligands are specific to eukaryotes; however, their genes have also been identified in the genomes of coralassociated bacteria, such as Endozoicomonas montiporae ${ }^{35}$. The ligands are thought to be involved in the infection process since they mediate several cellular functions, including endocytosis ${ }^{35}$. These observations suggest that the ephrin ligand is likely involved in Endozoicomonas infection.

We then successfully isolated Endozoicomonas species (Endozoicomonas sp. ISHI1) corresponding to OTU5 and analyzed its genome sequence. Subsequently, we identified eight ephrin ligand genes (e.g., NGGDMNLC_01928) (Supplementary Table 10) and found that their genomic locations were different from those in $E$. montiporae (Fig. 6d). These findings indicate that OTU5 acquired the host-like ephrin ligand genes at a different time than E. montiporae and that the ephrin ligand has an evolutionarily advantageous role for coral-bacteria interactions. 
We next performed a similarity search for the eight newly identified ephrin ligand genes using the NCBI nr database. The top hits were ephrin ligand genes from Acropora corals. The extracellular regions of the coral and Endozoicomonas ephrin ligand genes shared higher similarity (30.4\% identity) than those of the transmembrane regions (9.7\% identity) and cytoplasmic regions (23.3\% identity) (Fig. 6e). Hence, the extracellular domain might be important for establishment of the relationship between Endozoicomonas and the coral host.

\section{Discussion}

The knowledge of the interspecific interactions within a coral holobiont is limited because of the limitations of experimental methods that are currently available to investigate these interactions. Therefore, in the current study, we used a multi-omics approach to investigate the interactions between coral, zooxanthellae, and resident bacteria. We designed an efficient analysis pipeline and succeeded in determining the coral and zooxanthella gene expression profiles from data obtained by simultaneous sequencing of their transcripts. Then, using a systems biology approach, we successfully determined specific cross-species co-expression patterns between the coral host and zooxanthellae. We also described the inter-colony heterogeneity of the coral microbiome using $16 \mathrm{~S}$ rRNA amplicon sequencing. Integrated analysis of the coral transcriptome and microbiome revealed gene expression changes that correlated with the existence of coral-associated bacterial species and highlighted the ephrin genes as candidates involved in the coralbacterium crosstalk. This study demonstrates the potential of a data-driven approach for the analysis of cross-species interactions.

In the current study, the cross-species co-expression network analysis 
revealed that carbon fixation in zooxanthellae was positively correlated with coral host replication and negatively correlated with zooxanthella replication. This is consistent with previous reports suggesting that the doubling time of zooxanthellae during symbiosis is lower than that of zooxanthellae in culture ${ }^{7}$. The analysis also revealed that the replication process of zooxanthellae was negatively correlated with the phagocytosis and exocytosis of the coral host. Although several mechanisms of zooxanthella loss have been suggested, it is unclear which of these actually occur in nature ${ }^{6}$. The results presented herein suggest that the coral host actively regulates the number of intracellular algae by exocytosis-mediated expulsion and by degradation via phagocytosis. While the relationship between the coral and zooxanthellae has long been recognized as mutually beneficial, previous studies have challenged this notion and suggested that the relationship is dominated by coral host ${ }^{7}$. Similarly, the data-driven approach used in the current study revealed that fixed carbon is mainly used by the coral host, that the zooxanthella replication is suppressed during symbiosis, that zooxanthella density is actively maintained by the coral host, thus suggesting that the coral host presents a dominant interaction in the holobiont.

We also evaluated the inter-colony heterogeneity of coral microbiomes. Endozoicomonas dominated the microbiome of two colonies, Is 1 and Is2, while they were much less abundant in other colonies. Endozoicomonas have been predicted to have an intimate relationship with coral ${ }^{18}$, but their roles are mostly unknown and controversial ${ }^{13,17}$. In the current study, we showed that inflammation-related genes were upregulated in corals harboring Endozoicomonas species. The upregulated genes contained those involved in defense against bacterial infections, such as P2X7. Previous studies have reported that Endozoicomonas species are pathogenic 
to the fish host ${ }^{20,21}$. In addition, Endozoicomonas possess several eukaryotic-like genes like the E3 ubiquitin ligase gene, which have been identified in pathogenic bacteria such as Salmonella and Legionella ${ }^{36}$. Although a beneficial effect of Endozoicomonas has been previously proposed ${ }^{9,15}$, the results presented herein suggest that this is questionable, as the coral host exhibited a defense response against Endozoicomonas. Furthermore, the bleaching of colonies harboring Endozoicomonas, Is 1 and Is2, in the summer of 2016 was more severe than that of others colonies. Further verification of the beneficial effects of Endozoicomonas species for the coral host is necessary to resolve this.

While we have demonstrated here the potential of data-driven approaches, we acknowledge several limitations of this method. First, the presented analysis revealed that most zooxanthella transcripts were similar to those of Cladocopium, suggesting that Cladocopium was dominant in the sampled corals regardless of the sampling time and the coral host. However, we were unable to evaluate how the change in zooxanthella composition affected the results of the co-expression network analysis. Regarding this aspect of the current study, we believe that the approach will become more accurate as more zooxanthella genomes become available. Second, the presented analysis did not fully support the hypothesis that Endozoicomonas are beneficial to the coral host. However, we believe that further investigation will be necessary to confirm the presented results since we only detected the presence of Endozoicomonas in two of the colonies studied, with differences in the Endozoicomonas occurrence among colonies at the same sampling site. Using a large number of colonies from the same sampling site might be an effective strategy to implement in future studies.

In conclusion, we here proposed a data-driven approach for the investigation 
of cross-organism interactions involving coral, zooxanthellae, and bacteria. This approach is potentially applicable to diverse questions in coral biology. For example, it could be used to elucidate the association between bacterial infection and coral disease and the mechanism(s) of resistance to coral bleaching.

\section{Methods}

\section{Sample collection}

Coral branches were collected at three sampling sites [lshikawabaru (Is), Kyusanbashi $(K y)$, and Sesoko-minami $(M i)]$ around Sesoko Island, Japan, from November 2014 to September 2016, by scuba diving (Supplementary Fig. 1). Immediately after collection, the branches were soaked in RNAlater (Ambion, Austin, TX, USA), snap-frozen on ethanol with dry ice on the sampling ship, and stored at $80^{\circ} \mathrm{C}$ until RNA extraction. Before analysis, the branches were crushed and homogenized using an iron mortar and pestle, with the samples soaked in liquid nitrogen. Total RNA was extracted by using the RNeasy RNA extraction kit (QIAGEN, Hilden, Germany).

\section{Acquisition of environmental data}

Environmental parameters, including water temperature (Temp), pH, turbidity, salinity (Sal), solar intensity (Solar), photon flux (Quantum), water depth (Pressure), dissolved oxygen (ODO), fluorescent dissolved organic matter (fDOM), chlorophyll concentration (Chlorophyll), and phycocyanin of blue-green alge (BGA-PC), were measured at the sampling sites using YSI EXO2 water quality sonde (YSI Inc, Yellow Springs, OH, USA) and COMPACT-LW photon sensors (JFE Advantech, Nishinomiya, Japan) 


\section{Coral and zooxanthella transcriptome profiling}

Coral and zooxanthella mRNAs were obtained from total RNA, and sequencing libraries were prepared using TruSeq RNA Library Prep kit (Illumina, San Diego, CA, USA). The libraries were sequenced using the Illumina HiSeq 2000 platform (Illumina) in 100-bp paired-end mode. Low-quality reads were removed and adapters trimmed using bbduk in BBTools (sourceforge.net/projects/bbmap/) with the options "ktrim=r k=23 mink=11 hdist=1 tpe tbo qtrim=rl trimq=10 maq=10". The qualityfiltered reads were aligned with the genome of $A$. tenuis by HISAT2 ${ }^{37}$, followed by determination of coral expression profiles by StringTie ${ }^{38}$, as explained by Pertea et al $^{39}$. Unmapped reads were assembled using rnaSPAdes ${ }^{40}$, and contigs derived from zooxanthellae were identified by similarity search against the genome sequences of corals and zooxanthellae using BLASTn. The unmapped reads were aligned to the contigs to calculate zooxanthella expression profiles using RSEM ${ }^{41}$.

\section{Microbiome profiling by 16S rRNA amplicon sequencing}

The V1-V2 region of $16 \mathrm{~S}$ rRNA was amplified by RT-PCR using the primers PGM27F (CCATCTCATCCCTGCGTGTCTCCGACTCAG-[MID]GATAGAGTTTGATCMTGGCTCAG) and PGM-338R (CCTCTCTATGGGCAGTCGGTGATTGCTGCCTCCCGTAGGAGT) and sequenced using IonPGM (Thermo Fisher Scientific, Waltham, MA, USA) to profile bacterial community structure. The sequence reads were preprocessed as follows: (i) the regions corresponding to PCR primers were trimmed using TagCleaner ${ }^{42}$; (ii) the reads expected to contain $>1 \%$ errors were removed with $U S E A R C H{ }^{43}$; and (iii) the reads shorter than $270 \mathrm{bp}$ were excluded. The preprocessed reads were truncated to 270 bp and clustered into OTUs using UPARSE ${ }^{44}$. OTUs were taxonomically 
classified using RDP Classifier ${ }^{45}$, and were designated as "undetermined" if their bootstrap values were below 80. A phylogenetic tree for the OTUs was constructed using FastTree ${ }^{46}$ after multiple alignments with MAFFT ${ }^{47}$. UniFrac analysis, based on the constructed tree, was performed using the R package phyloseq ${ }^{48}$.

\section{Determination of zooxanthella cell density}

To calculate zooxanthella cell density in coral branches (cells per square centimeter), the number of zooxanthella cells in coral tissue homogenates and the surface area of the corresponding coral skeleton with the tissue completely removed were determined. Homogenates of $A$. tenuis branches were prepared using AirFloss (Philips, Amsterdam, Netherlands) filled with $\mathrm{Mg}^{2+}$ - and $\mathrm{Ca}^{2+}$-free artificial seawater ( $\mathrm{NaCl}, 401.8 \mathrm{mM} ; \mathrm{Na}_{2} \mathrm{SO}_{4}, 27.6 \mathrm{mM} ; \mathrm{Na}_{2} \mathrm{HCO}_{3}, 2.29 \mathrm{mM} ; \mathrm{KCl}, 8.91 \mathrm{mM} ; \mathrm{KBr}, 0.81$ $\mathrm{mM}$; NaF, $0.62 \mathrm{mM} ; \mathrm{H}_{3} \mathrm{BO}_{4}, 0.39 \mathrm{mM} ; \mathrm{SrCl}_{2}, 11.3 \mu \mathrm{M}$; EDTA-Na $2,20 \mathrm{mM}$ ). The homogenate volume was determined, and zooxanthella cells were counted in a small portion of the homogenate using a $20 \mu \mathrm{m}$-depth hemocytometer. Surface area of the coral skeleton was measured as described previously ${ }^{49}$.

\section{Genome assembly of Endozoicomonas isolate}

Coral branches were rinsed in sterilized PBSE (phosphate-buffered saline with 20 mM EDTA). The tissue was then picked off using a sterilized toothpick, plated on marine broth supplemented with cycloheximide $(100 \mathrm{~g} / \mathrm{mL})$, and incubated at $27^{\circ} \mathrm{C}$ for 2 weeks. The obtained bacterial isolate was identified as Endozoicomonas species OTU5 by Sanger sequencing of 16S rRNA gene, and was grown in marine broth at $27^{\circ} \mathrm{C}$ for additional 2 weeks. Genomic DNA was extracted by using the DNeasy Plant Mini Kit (QIAGEN), and pair-end libraries were prepared using the 
Nextera Library Prep Kit (Illumina) and sequenced using the Illumina MiSeq platform (Illumina). Low-quality reads were removed, and sequence adaptors were trimmed using fastp ${ }^{50}$. Genome assembly of the quality-filtered sequence reads was conducted using SPAdes ${ }^{51}$ in "careful" mode.

\section{Genome analysis of Endozoicomonas isolate}

Publicly available genome sequences of 10 Endozoicomonas strains were downloaded from RefSeq (ftp://ftp.ncbi.nlm.nih.gov/genomes/refseq/bacteria/) for comparative genome analysis. Gene regions in each genome were predicted by using Prokka ${ }^{52}$, and their functions were annotated using KAAS ${ }^{53}$. Similar genes were grouped into orthogroups using Orthofinder ${ }^{54}$. The genomes were aligned by using progressiveMauve ${ }^{55}$ to define locally collinear blocks (LCBs), i.e., conserved genome regions among strains.

\section{Statistics}

\section{Co-expression network analysis}

Genetic modules in corals and zooxanthellae were determined based on weighted gene co-expression network analysis using the R package WGCNA ${ }^{45}$. Genes expressed in $<50 \%$ of the samples were excluded. The power parameter (coral: 14 ; zooxanthellae: 23) was selected to achieve good fitness $\left(R^{2}>0.8\right)$ for scale-free topology of the co-expression network.

\section{Gene set analysis}

Functional annotation of the transcripts was performed using two methods. (i) Uniprot IDs were assigned to the transcripts by BLASTx search against the 
Swiss?Prot database. UniProt IDs of the top hits were converted to gene ontology

(GO) terms. (ii) KEGG orthology (KO) IDs were assigned to the gene clusters using KAAS $^{53}$. Gene set analysis was performed by Fisher's exact test using the $\mathrm{R}$ package ClusterProfiler ${ }^{56}$.

\section{Data availability}

Raw RNA-seq reads and 16S rRNA amplicon sequences were deposited in NCBI under the BioProject accession numbers PRJNA743235 and PRJNA742893. The genome sequence of Endozoicomonas sp. ISHI1 is available at NCBI Genome under the accession number JAGRPU000000000.

\section{Code availability}

The source codes and details of the analyses performed in this paper are available at https://tmaruy.github.io/coral_multi_omics.

\section{References}

1. Yellowlees, D., Rees, T. A. V. \& Leggat, W. Metabolic interactions between algal symbionts and invertebrate hosts. Plant Cell Environ. 31, 679-694 (2008).

2. Davy, S. K., Allemand, D. \& Weis, V. M. Cell biology of cnidarian-dinoflagellate symbiosis. Microbiol. Mol. Biol. Rev. 76, 229-261 (2012).

3. T. Banaszak, A., LaJeunesse, T. C. \& Trench, R. K. The synthesis of mycosporine-like amino acids (MAAs) by cultured, symbiotic dinoflagellates. J. Exp. Mar. Bio. Ecol. 249, 219-233 (2000).

4. Shinzato, C. et al. Using the Acropora digitifera genome to understand coral responses to environmental change. Nature 476, 320-323 (2011).

5. Loya, Y. et al. Coral bleaching: the winners and the losers. Ecol. Lett. 4, 122-131 
(2001).

6. Weis, V. M. Cellular mechanisms of Cnidarian bleaching: stress causes the collapse of symbiosis. J. Exp. Biol. 211, 3059-3066 (2008).

7. Wooldridge, S. A. Is the coral $\square$ algae symbiosis really 'mutually beneficial'for the partners? Bioessays (2010).

8. Reshef, L., Koren, O., Loya, Y., Zilber-Rosenberg, I. \& Rosenberg, E. The coral probiotic hypothesis. Environ. Microbiol. 8, 2068-2073 (2006).

9. Bourne, D., lida, Y., Uthicke, S. \& Smith-Keune, C. Changes in coral-associated microbial communities during a bleaching event. ISME J. 2, 350-363 (2008).

10. Closek, C. J. et al. Coral transcriptome and bacterial community profiles reveal distinct Yellow Band Disease states in Orbicella faveolata. ISME J. 8, 2411-2422 (2014).

11. Cárdenas, A., Rodriguez-R, L. M., Pizarro, V., Cadavid, L. F. \& Arévalo-Ferro, C. Shifts in bacterial communities of two Caribbean reef-building coral species affected by white plague disease. ISME J. 6, 502-512 (2012).

12. Garcia, G. D. et al. Metagenomic analysis of healthy and white plague-affected Mussismilia braziliensis corals. Microb. Ecol. 65, 1076-1086 (2013).

13. Meyer, J. L., Paul, V. J. \& Teplitski, M. Community shifts in the surface microbiomes of the coral Porites astreoides with unusual lesions. PLoS One 9, e100316 (2014).

14. Ziegler, M., Seneca, F. O., Yum, L. K., Palumbi, S. R. \& Voolstra, C. R. Bacterial community dynamics are linked to patterns of coral heat tolerance. Nat. Commun. $\mathbf{8}$, 14213 (2017).

15. Glasl, B., Herndl, G. J. \& Frade, P. R. The microbiome of coral surface mucus has a key role in mediating holobiont health and survival upon disturbance. ISME J. 10, 22802292 (2016).

16. Sunagawa, S., Woodley, C. M. \& Medina, M. Threatened corals provide underexplored microbial habitats. PLoS One 5, e9554 (2010).

17. Neave, M. J., Apprill, A., Ferrier-Pagès, C. \& Voolstra, C. R. Diversity and function of prevalent symbiotic marine bacteria in the genus Endozoicomonas. Appl. Microbiol. 
Biotechnol. 100, 8315-8324 (2016).

18. Bayer, T. et al. The microbiome of the Red Sea coral Stylophora pistillata is dominated by tissue-associated Endozoicomonas bacteria. Appl. Environ. Microbiol. 79, 47594762 (2013).

19. Neave, M. J. et al. Differential specificity between closely related corals and abundant Endozoicomonas endosymbionts across global scales. ISME J. 11, 186-200 (2017).

20. Mendoza, M. et al. A novel agent (Endozoicomonas elysicola) responsible for epitheliocystis in cobia Rachycentrum canadum larvae. Dis. Aquat. Organ. 106, 31-37 (2013).

21. Katharios, P. et al. Environmental marine pathogen isolation using mesocosm culture of sharpsnout seabream: striking genomic and morphological features of novel Endozoicomonas sp. Sci. Rep. 5, 17609 (2015).

22. Krueger, T. \& Gates, R. D. Cultivating endosymbionts-Host environmental mimics support the survival of Symbiodinium C15 ex hospite. J. Exp. Mar. Bio. Ecol. (2012).

23. Stewart, E. J. Growing unculturable bacteria. J. Bacteriol. 194, 4151-4160 (2012).

24. Westermann, A. J. \& Vogel, J. Cross-species RNA-seq for deciphering host-microbe interactions. Nat. Rev. Genet. 1-18 (2021).

25. Shinzato, C., Inoue, M. \& Kusakabe, M. A snapshot of a coral 'holobiont': a transcriptome assembly of the scleractinian coral, porites, captures a wide variety of genes from both the host and symbiotic zooxanthellae. PLoS One 9, e85182 (2014).

26. Shinzato, C. et al. Eighteen coral genomes reveal the evolutionary origin of Acropora strategies to accommodate environmental changes. Mol. Biol. Evol. (2020) doi:10.1093/molbev/msaa216.

27. Shoguchi, E. et al. Draft assembly of the Symbiodinium minutum nuclear genome reveals dinoflagellate gene structure. Curr. Biol. 23, 1399-1408 (2013).

28. Lin, S. et al. The Symbiodinium kawagutii genome illuminates dinoflagellate gene expression and coral symbiosis. Science 350, 691-694 (2015).

29. The ReFuGe 2020 Consortium - using 'omics' approaches to explore the adaptability 
and resilience of coral holobionts to environmental change. Front. Mar. Sci. 2, (2015).

30. LaJeunesse, T. C. et al. Systematic Revision of Symbiodiniaceae Highlights the Antiquity and Diversity of Coral Endosymbionts. Curr. Biol. 28, 2570-2580.e6 (2018).

31. Ide, K. et al. High-Quality Draft Genome Sequence of a Rickettsiales Bacterium Found in Acropora tenuis Coral from Okinawa, Japan. Microbiol Resour Announc 9, (2020).

32. Klinges, G., Maher, R. L., Vega Thurber, R. L. \& Muller, E. M. Parasitic ' Candidatus Aquarickettsia rohweri' is a marker of disease susceptibility in Acropora cervicornis but is lost during thermal stress. Environmental Microbiology (2020) doi:10.1111/14622920.15245.

33. Di Virgilio, F., Dal Ben, D., Sarti, A. C., Giuliani, A. L. \& Falzoni, S. The P2X7 Receptor in Infection and Inflammation. Immunity 47, 15-31 (2017).

34. Rump, A., Smolander, O. P., Rüütel Boudinot, S., Kanellopoulos, J. M. \& Boudinot, P. Evolutionary Origin of the P2X7 C-ter Region: Capture of an Ancient Ballast Domain by a P2X4-Like Gene in Ancient Jawed Vertebrates. Front. Immunol. 11, 113 (2020).

35. Ding, J.-Y., Shiu, J.-H., Chen, W.-M., Chiang, Y.-R. \& Tang, S.-L. Genomic Insight into the Host-Endosymbiont Relationship of Endozoicomonas montiporae CL-33T with its Coral Host. Front. Microbiol. 7, 251 (2016).

36. Maculins, T., Fiskin, E., Bhogaraju, S. \& Dikic, I. Bacteria-host relationship: ubiquitin ligases as weapons of invasion. Cell Res. 26, 499-510 (2016).

37. Kim, D., Paggi, J. M., Park, C., Bennett, C. \& Salzberg, S. L. Graph-based genome alignment and genotyping with HISAT2 and HISAT-genotype. Nat. Biotechnol. 37, 907915 (2019).

38. Pertea, M. et al. StringTie enables improved reconstruction of a transcriptome from RNA-seq reads. Nat. Biotechnol. 33, 290-295 (2015).

39. Pertea, M., Kim, D., Pertea, G. M., Leek, J. T. \& Salzberg, S. L. Transcript-level expression analysis of RNA-seq experiments with HISAT, StringTie and Ballgown. Nat. Protoc. 11, 1650-1667 (2016).

40. Bushmanova, E., Antipov, D., Lapidus, A. \& Prjibelski, A. D. rnaSPAdes: a de novo 
transcriptome assembler and its application to RNA-Seq data. Gigascience 8, (2019).

41. Li, B. \& Dewey, C. N. RSEM: accurate transcript quantification from RNA-Seq data with or without a reference genome. BMC Bioinformatics 12, 323 (2011).

42. Schmieder, R., Lim, Y. W., Rohwer, F. \& Edwards, R. TagCleaner: Identification and removal of tag sequences from genomic and metagenomic datasets. $B M C$ Bioinformatics 11, 341 (2010).

43. Edgar, R. C. Search and clustering orders of magnitude faster than BLAST. Bioinformatics 26, 2460-2461 (2010).

44. Edgar, R. C. UPARSE: highly accurate OTU sequences from microbial amplicon reads. Nat. Methods 10, 996-998 (2013).

45. Wang, Q., Garrity, G. M., Tiedje, J. M. \& Cole, J. R. Naive Bayesian classifier for rapid assignment of rRNA sequences into the new bacterial taxonomy. Appl. Environ. Microbiol. 73, 5261-5267 (2007).

46. Price, M. N., Dehal, P. S. \& Arkin, A. P. FastTree 2--approximately maximum-likelihood trees for large alignments. PLoS One 5, e9490 (2010).

47. Katoh, K., Misawa, K., Kuma, K.-I. \& Miyata, T. MAFFT: a novel method for rapid multiple sequence alignment based on fast Fourier transform. Nucleic Acids Res. 30, 3059-3066 (2002).

48. McMurdie, P. J. \& Holmes, S. phyloseq: an R package for reproducible interactive analysis and graphics of microbiome census data. PLoS One (2013).

49. Marsh, J. A., Jr. Primary productivity of reef-building calcareous red algae. Ecology 51, 255-263 (1970).

50. Chen, S., Zhou, Y., Chen, Y. \& Gu, J. fastp: an ultra-fast all-in-one FASTQ preprocessor. Bioinformatics 34, i884-i890 (2018).

51. Bankevich, A. et al. SPAdes: a new genome assembly algorithm and its applications to single-cell sequencing. J. Comput. Biol. 19, 455-477 (2012).

52. Seemann, T. Prokka: rapid prokaryotic genome annotation. Bioinformatics 30, 20682069 (2014). 
53. Moriya, Y., Itoh, M., Okuda, S., Yoshizawa, A. C. \& Kanehisa, M. KAAS: an automatic genome annotation and pathway reconstruction server. Nucleic Acids Res. 35, W182-5 (2007).

54. Emms, D. M. \& Kelly, S. OrthoFinder: phylogenetic orthology inference for comparative genomics. Genome Biol. 20, 238 (2019).

55. Darling, A. E., Mau, B. \& Perna, N. T. progressiveMauve: multiple genome alignment with gene gain, loss and rearrangement. PLoS One 5, e11147 (2010).

56. Yu, G., Wang, L.-G., Han, Y. \& He, Q.-Y. clusterProfiler: an R Package for Comparing Biological Themes Among Gene Clusters. OMICS 16, 284-287 (2012). 


\section{Acknowledgements}

This work was supported by JST-CREST (grant number JPMJCR12A4). The supercomputing resource was provided by the Human Genome Center (the University of Tokyo, Tokyo, Japan).

\section{Author contributions}

TM analyzed data. MI, SW, YO, TM and YNi collected the samples and sequenced them. $\mathrm{MI}, \mathrm{YO}, \mathrm{YNi}$, and $\mathrm{KI}$ isolated the bacteria and sequenced the bacterial genome. HF, SS, and YNa performed environmental monitoring. HT, CS, NS, and KY guided the research. TM and $\mathrm{HT}$ wrote the manuscript.

\section{Competing interests}

The authors declare no competing interests.

\section{Materials and Correspondence}

Correspondence to Haruko Takeyama.

\section{Figure legends}

Fig. 1 Multi-omics profiling of the coral holobiont. a Overview of experimental procedures for acquiring multi-omics datasets. $\mathbf{b}$ Bioinformatics pipeline for multiomics analysis of the coral holobiont.

Fig. 2 Season- and colony-dependent patterns of coral gene expression. a,b Module eigengenes (the expression level of modules) of season-dependent modules (a) and colony-dependent modules (b). c,d Correlation between environmental 
parameters and module eigengenes. Statistical significance of the correlation is denoted as follows: ${ }^{*} p<0.05,{ }^{* *} p<0.01,{ }^{* * *} p<0.00001$.

\section{Fig. 3 Season- and colony-dependent patterns of zooxanthella gene}

expression. a,b Module eigengenes of season-dependent modules (a) and colonydependent modules (b). c,d Correlation between environmental parameters and module eigengenes. Statistical significance of the correlation is denoted as follows: * $p<0.05,{ }^{* *} p<0.01,{ }^{* * *} p<0.00001$.

Fig. 4 Cross-species co-expression network of the crosstalk between coral and symbiotic algae. Co-expression network created using the WGCNA modules.

Circles and diamonds correspond to the modules of coral and symbiotic algae, respectively. Orange and blue edges represent positive and negative correlations of the module eigengenes, respectively.

Fig. 5 Composition of the coral microbiome. a,b Composition of the coral microbiome at the genus level (a) and OTU level (b). Genera and OTUs with an abundance of less than $10 \%$ in all samples are denoted as "Others". OTU genera were based on top hits after similarity search against the SILVA database. c Embedding of the community structure by principal coordinate analysis (PCoA) using unweighted UniFrac distance. d-f Proportions of Cyanobacteria GpVIII, Anaplasma, and Endozoicomonas in each colony. g Proportions of four major Endozoicomonas OTUs.

Fig. 6 Association between coral gene expression and the microbiome. a,b Genes differentially expressed in colonies harboring Endozoicomonas (Is1, Is2): 
$P 2 R X 7(\mathbf{a})$ and ephrin ligand genes (b). c Locally collinear block (LCB) around ephrin-B2 genes in OTU5. Genes are colored by orthogroups defined with Orthofinder. d Multiple alignment of ephrin ligand genes in coral (A. tenuis), Endozoicomonas (OTU5), and human. e Similarity between the ephrin genes of OTU5 and $A$. tenuis.

Supplementary Fig. 1 Sampling site. Scleractinian corals Acropora tenuis were sampled at the three sampling sites $(I s, K y, M i)$ around Sesoko island, Okinawa, Japan.

Supplementary Fig. 2 Changes in zooxanthellae densities over time. a Seasonal change of zooxanthellae densities in each coral colony. b Representative photos of Is2, a colony which showed prominent bleaching in 2016 Aug and 2016 Sep.

\section{Supplementary Fig. 3 De novo transcriptome assembly using unmapped reads.}

a Proportion of the contigs derived from coral and zooxanthellae. Contigs of coral and zooxanthellae were classified by similarity search against transcripts of $A$. tenuis and zooxanthellae. Contigs showing no similarity with both transcripts were represented as unclassified. b GC contents of the contigs. c Length of the contigs.

\section{Supplementary Fig. 4 Function of temperature-dependent modules in corals.}

Dot plots depict gene ontology (GO) terms and KEGG pathways significantly associated with the temperature-dependent modules. Colors and sizes of the dots correspond to adjusted $p$ values and proportion of genes belonging to the functional groups in the modules. Statistical significance was calculated by Fisher's exact test. 
Supplementary Fig. 5 Size and annotation rate of the modules. Bar plots represent module size, proportion of genes showing similarities with sequences in Swissprot and KEGG.

\section{Supplementary Fig. 6 Function of colony-dependent modules in corals. Dot}

plots depict gene ontology (GO) terms and KEGG pathways significantly associated with the colony-dependent modules. Colors and sizes of the dots correspond to adjusted p-values and proportion of genes belonging to the functional groups in the modules. Statistical significance was calculated by Fisher's exact test.

\section{Supplementary Fig. 7 Taxonomic assignment for zooxanthellae transcripts. Bar} chart depicts the taxonomic composition of zooxanthellae transcripts. Similarity searches against protein sequences of six zooxanthellae species were conducted. Each transcript was classified based on the origin of the best hit sequence. Symbiodinium sp. clade A3, $S$. microadriaticum (clade A), Breviolum minutum (clade B), Cladocopium sp. clade C, C. goreaui (clade C1) and Fugacium kawagutii (clade F).

\section{Fig. S8 Similarities of coral transcriptome, zooxanthellae transcriptome and} microbiome among samples. $\mathbf{a}-\mathbf{c}$ Similarities of $\mathbf{a}$ coral transcriptome, $\mathbf{b}$ zooxanthellae transcriptome and $\mathbf{c}$ microbiome among samples. Pearson's correlation coefficient was used as a similarity indicator for transcriptome of coral and zooxanthellae. Weighted UniFrac distance was used for microbiomes. d-f Box plot of the similarities. Midlines in the box represent medians. Upper and lower hinges represent $25 \%$ quantiles and $75 \%$ quantiles. Upper and lower whiskers represent maximum and minimum values. 
bioRxiv preprint doi: https://doi.org/10.1101/2021.10.25.465660; this version posted October 26, 2021. The copyright holder for this preprint (which was not certified by peer review) is the author/funder. All rights reserved. No reuse allowed without permission.

\section{Figures}

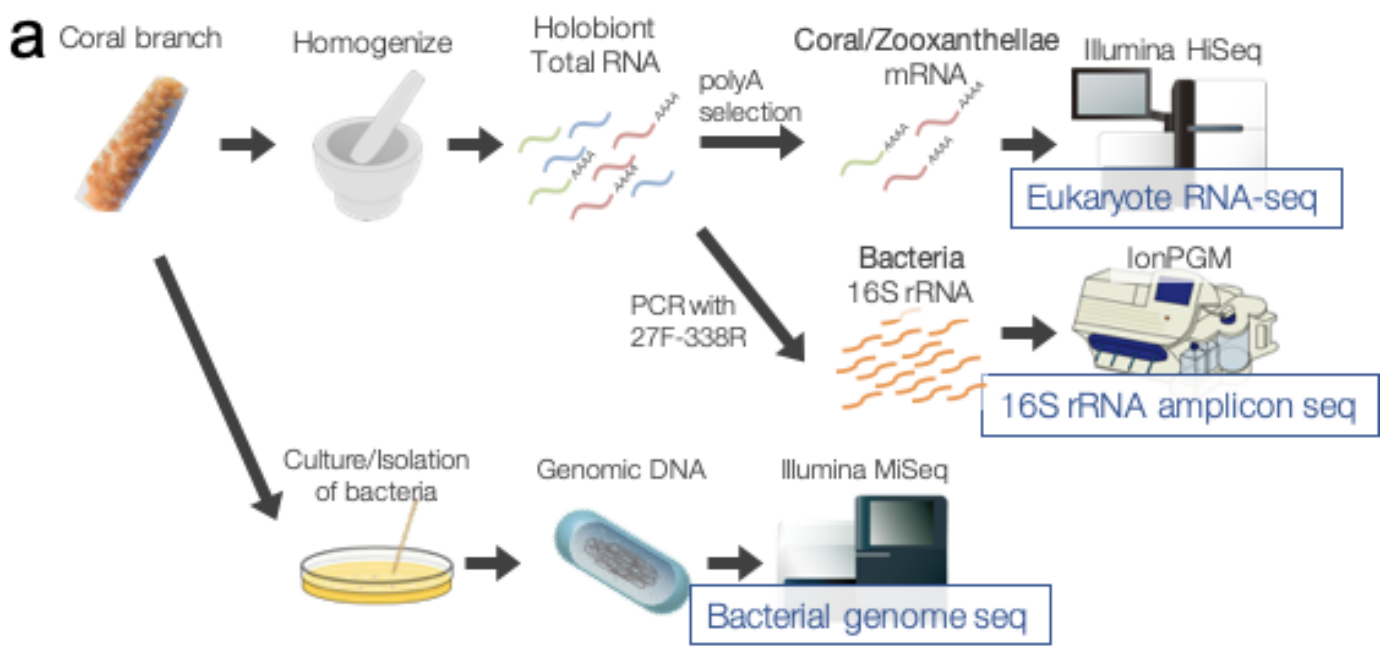

b

\section{Eukaryote RNA-seg}

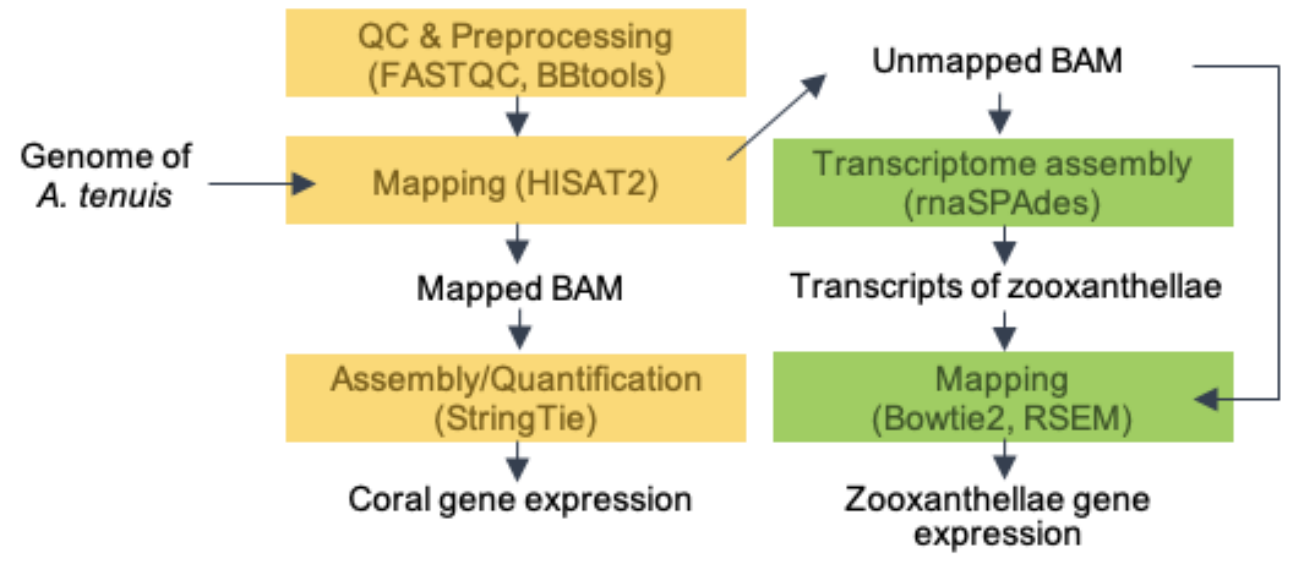

Figure 1 
bioRxiv preprint doi: https://doi.org/10.1101/2021.10.25.465660; this version posted October 26, 2021. The copyright holder for this preprint (which was not certified by peer review) is the author/funder. All rights reserved. No reuse allowed without permission.
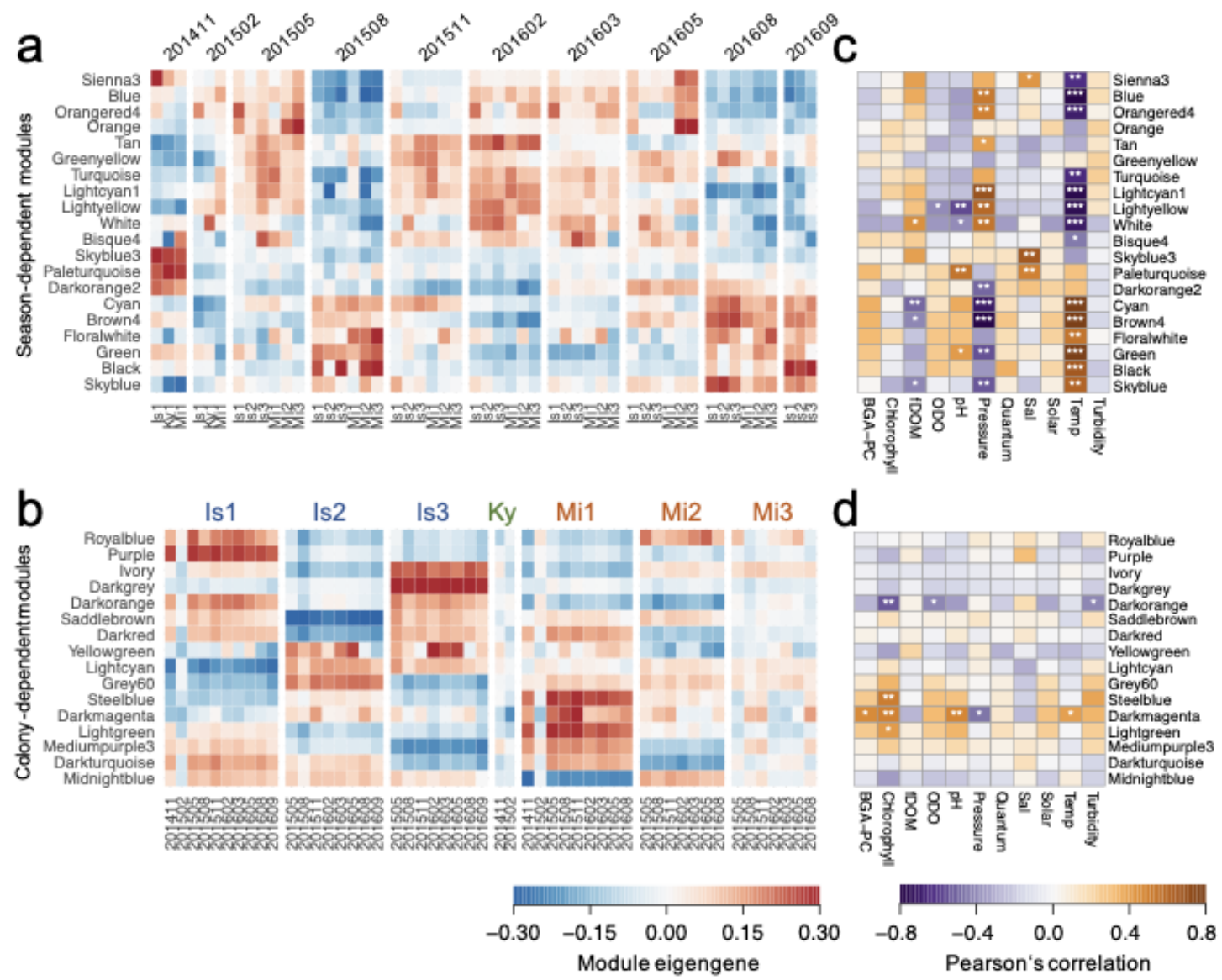

Figure 2 
bioRxiv preprint doi: https://doi.org/10.1101/2021.10.25.465660; this version posted October 26, 2021. The copyright holder for this preprint (which was not certified by peer review) is the author/funder. All rights reserved. No reuse allowed without permission.
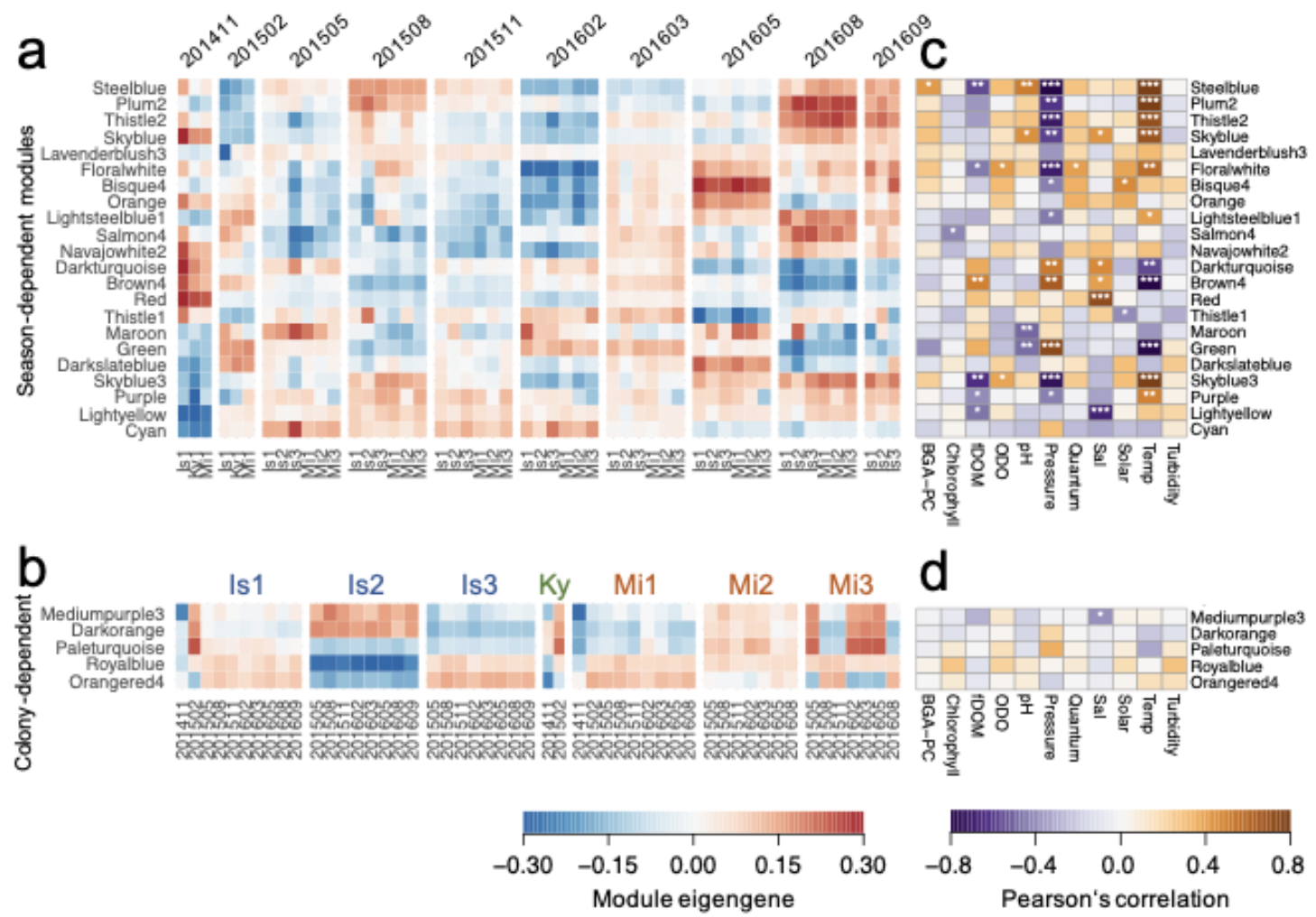

Figure 3 
bioRxiv preprint doi: https://doi.org/10.1101/2021.10.25.465660; this version posted October 26, 2021. The copyright holder for this preprint (which was not certified by peer review) is the author/funder. All rights reserved. No reuse allowed without permission.

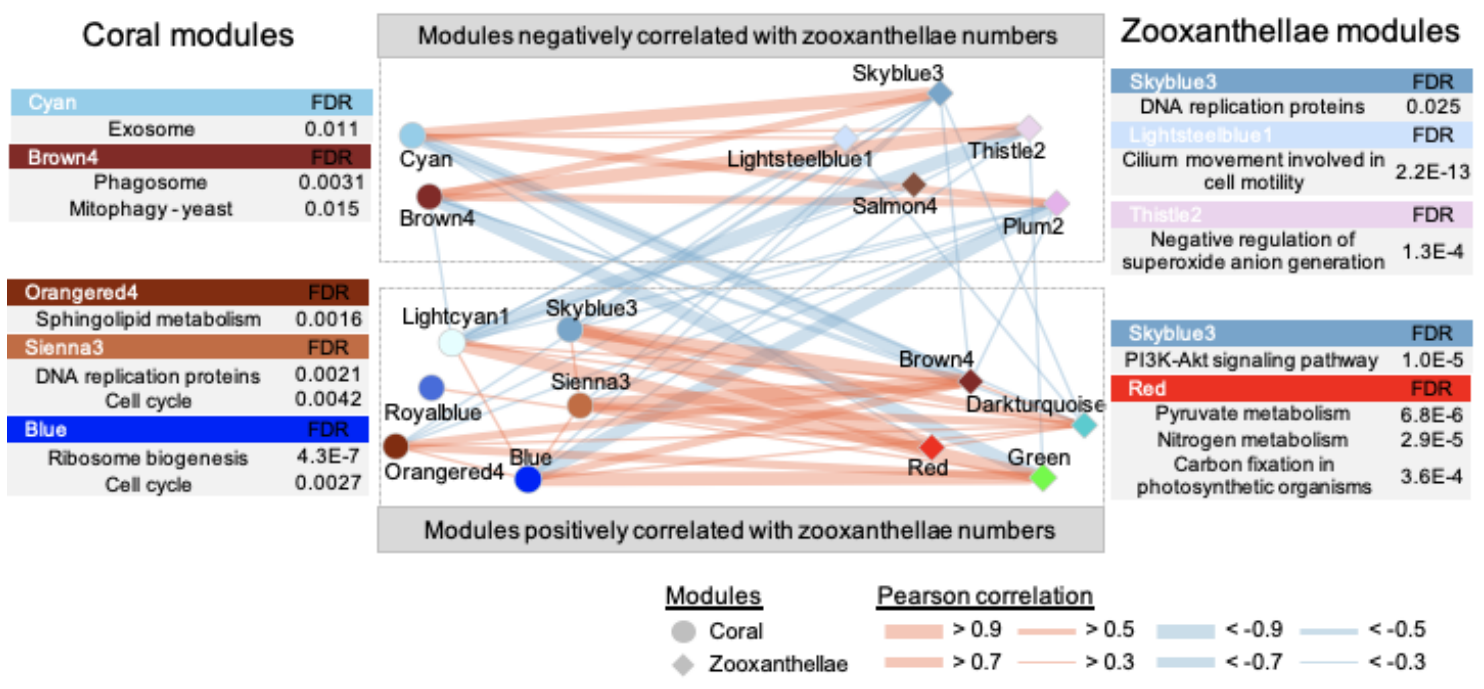

Figure 4 
bioRxiv preprint doi: https://doi.org/10.1101/2021.10.25.465660; this version posted October 26, 2021. The copyright holder for this preprint (which was not certified by peer review) is the author/funder. All rights reserved. No reuse allowed without permission.

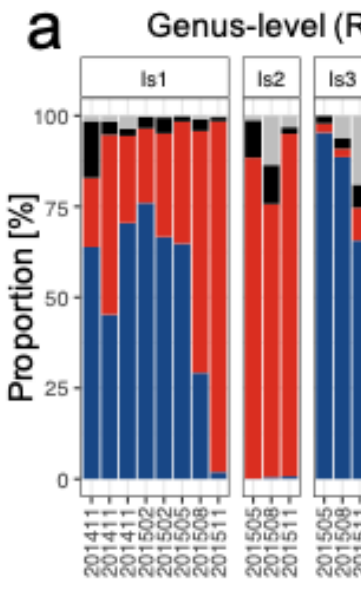

Anaplasma

Endozoicomonas

Cyanobacteria GpVIII

Unclassified

Others

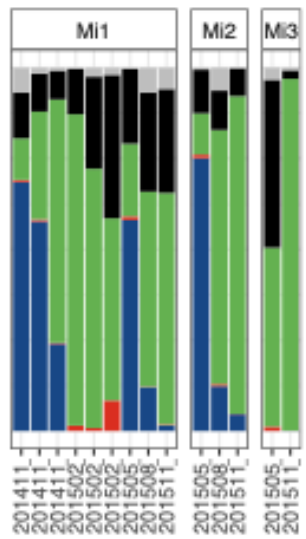

b

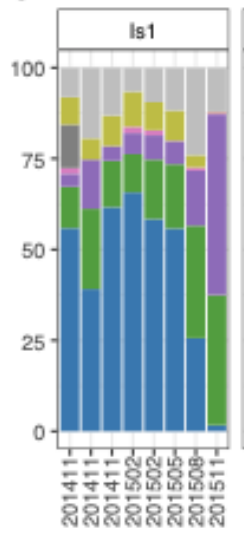

OTU-level

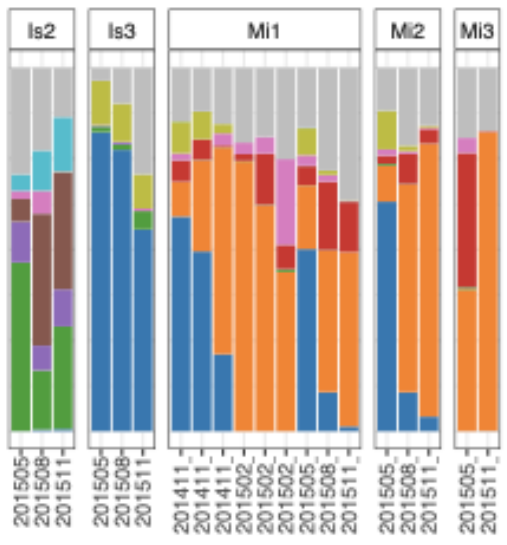

C Unweighted UniFrac

OTU1 Anaplasma phagocytophilum $84.4 \%$ OTU2 Pantanalinema rosaneae $\quad 91.5 \%$ OTU3 Endozoicomonas gorgoniicola $88.9 \%$

OTU4 Iphinoe spelaeobios

OTU5 Endozoicomonas gorgoniicola $91.7 \%$
OTU13 Endozoicomonas gorgonilicola $89.9 \%$ OTU14 Unclassified

OTU16 Sphingomonas phyllosphaerae $80.5 \%$ OTU40 Anaplasma phagocytophilum $80.9 \%$ OTU549 Endozoicomonas gorgoniicola $88.2 \%$
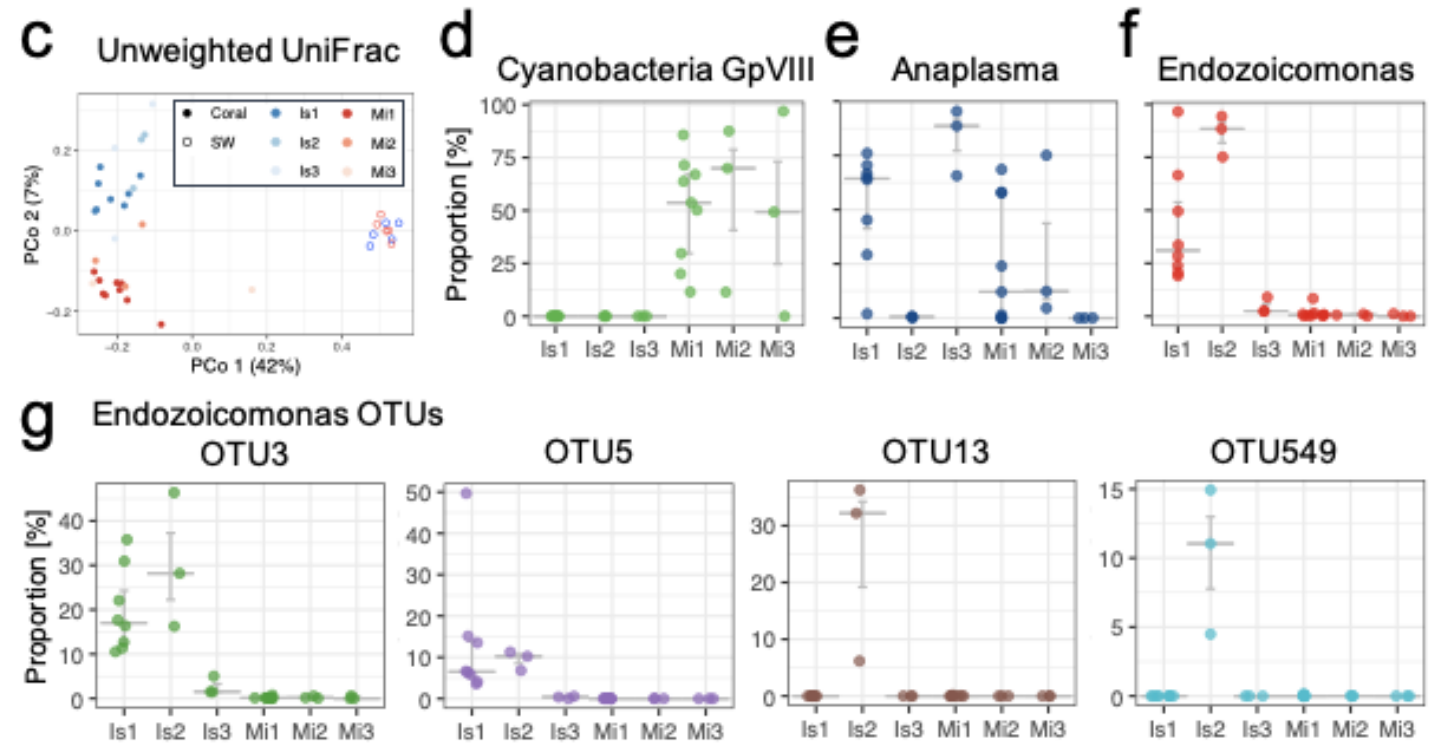

Figure 5 
bioRxiv preprint doi: https://doi.org/10.1101/2021.10.25.465660; this version posted October 26, 2021. The copyright holder for this preprint (which was not certified by peer review) is the author/funder. All rights reserved. No reuse allowed without permission.

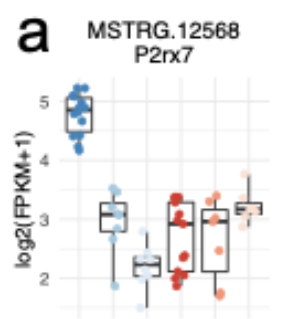

Is1 ls:2 Is3 Mi1 Mi2 Mi3
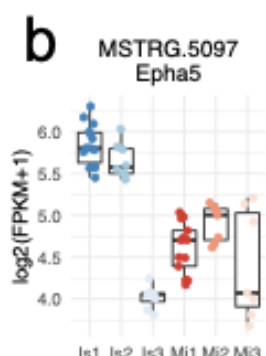

Is1 ls2 is Mi1 Mi2M:

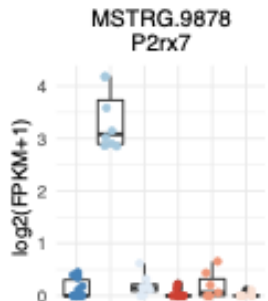

Is1 ls2 is3 Mi1 M2 Mis

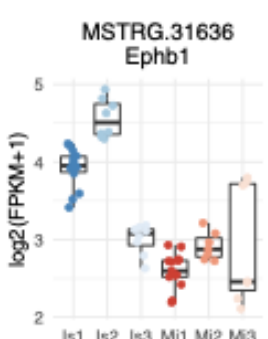

C
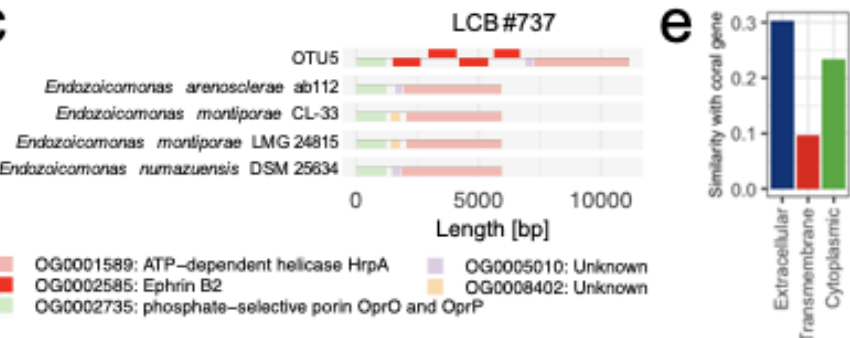

d
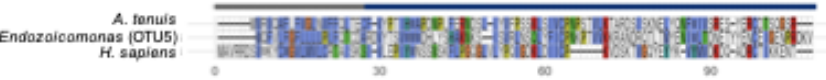

A. fonuis

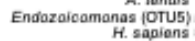

H. sapiens
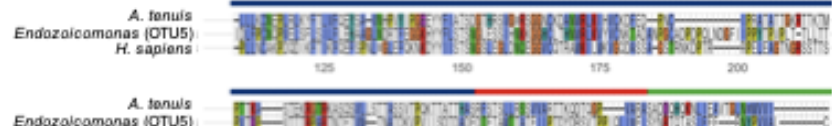

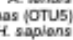

tom

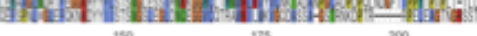

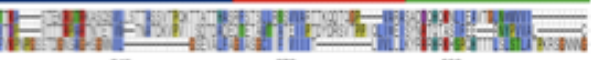

A. tanuis
Endazalcamanas (107u5)
H. sapians

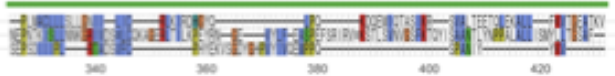

Extracellular $\longrightarrow$ Transmembrane $=$ Cytoplasmic region

Figure 6 
bioRxiv preprint doi: https://doi.org/10.1101/2021.10.25.465660; this version posted October 26, 2021. The copyright holder for this preprint (which was not certified by peer review) is the author/funder. All rights reserved. No reuse allowed without permission.
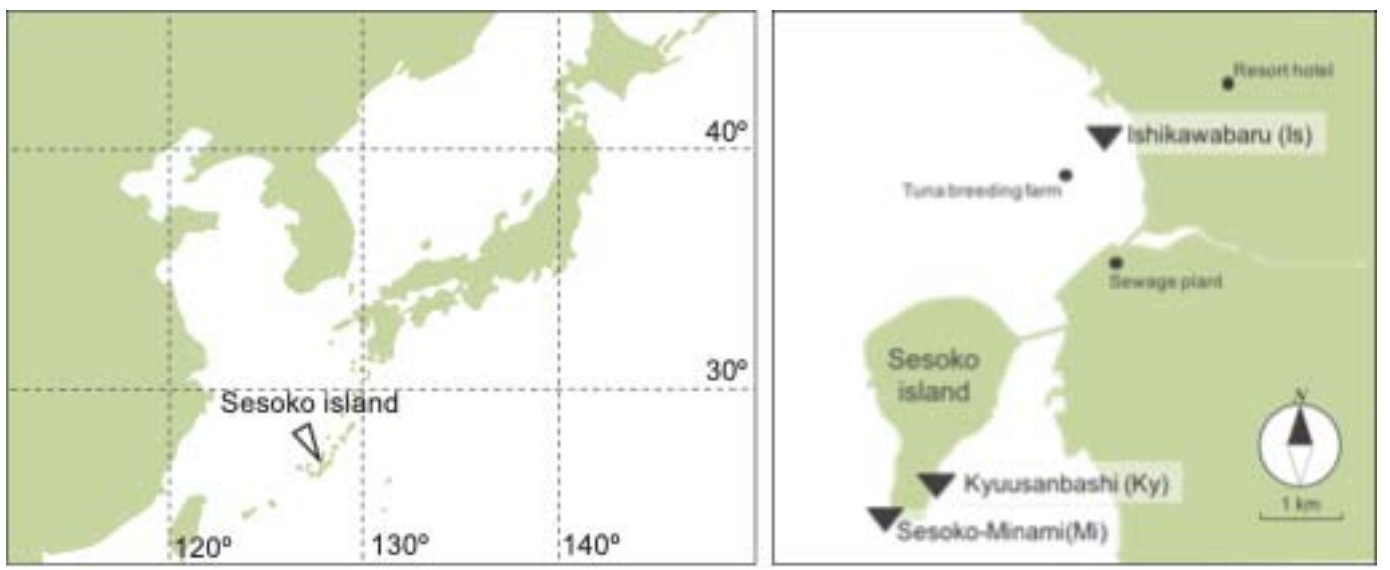

\section{Supplementary Figure 1}


bioRxiv preprint doi: https://doi.org/10.1101/2021.10.25.465660; this version posted October 26, 2021. The copyright holder for this preprint (which was not certified by peer review) is the author/funder. All rights reserved. No reuse allowed without permission.

a

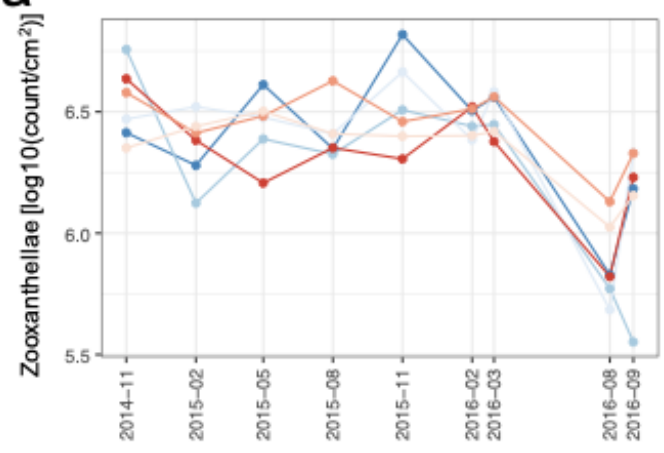

b Photos of colony Is2

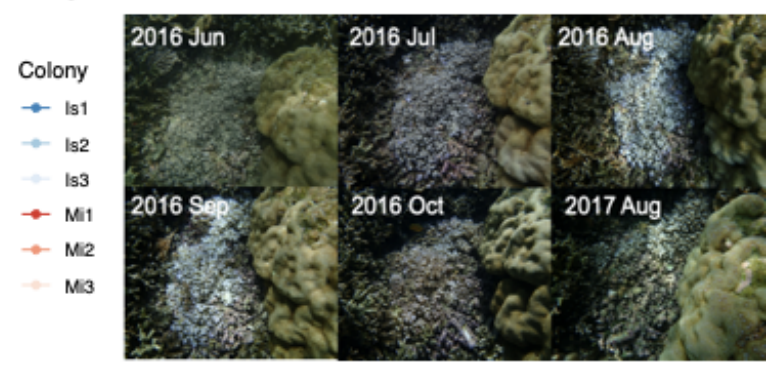

\section{Supplementary Figure 2}


bioRxiv preprint doi: https://doi.org/10.1101/2021.10.25.465660; this version posted October 26, 2021. The copyright holder for this preprint (which was not certified by peer review) is the author/funder. All rights reserved. No reuse allowed without permission.
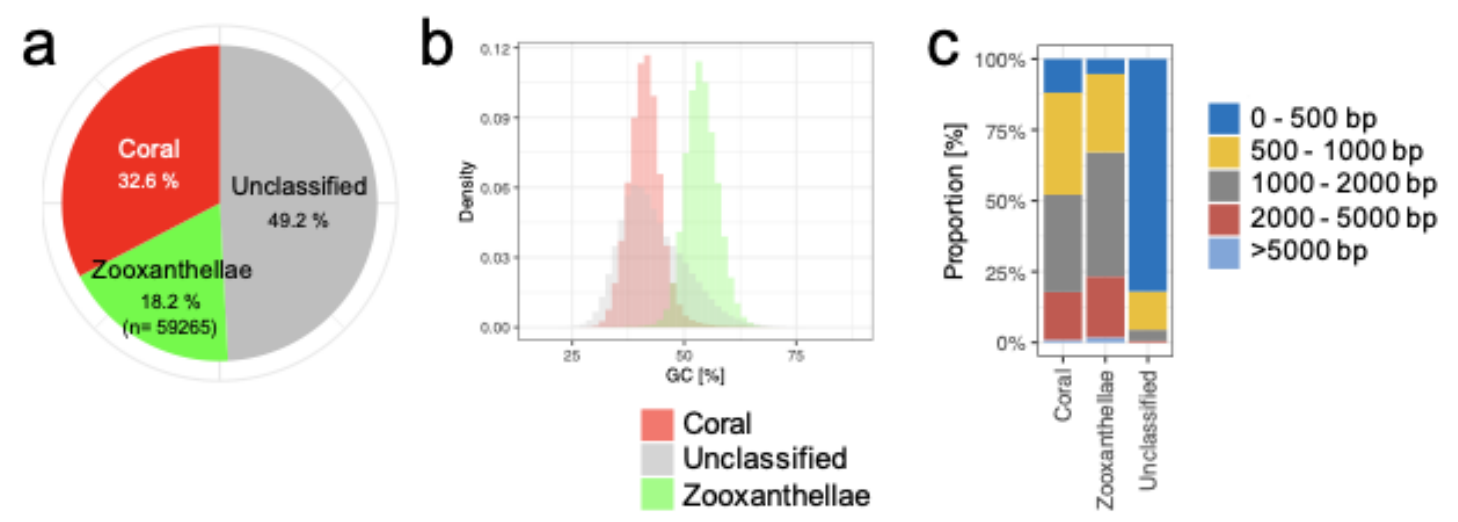

\section{Supplementary Figure 3}


bioRxiv preprint doi: https://doi.org/10.1101/2021.10.25.465660; this version posted October 26, 2021. The copyright holder for this preprint (which was not certified by peer review) is the author/funder. All rights reserved. No reuse allowed without permission.
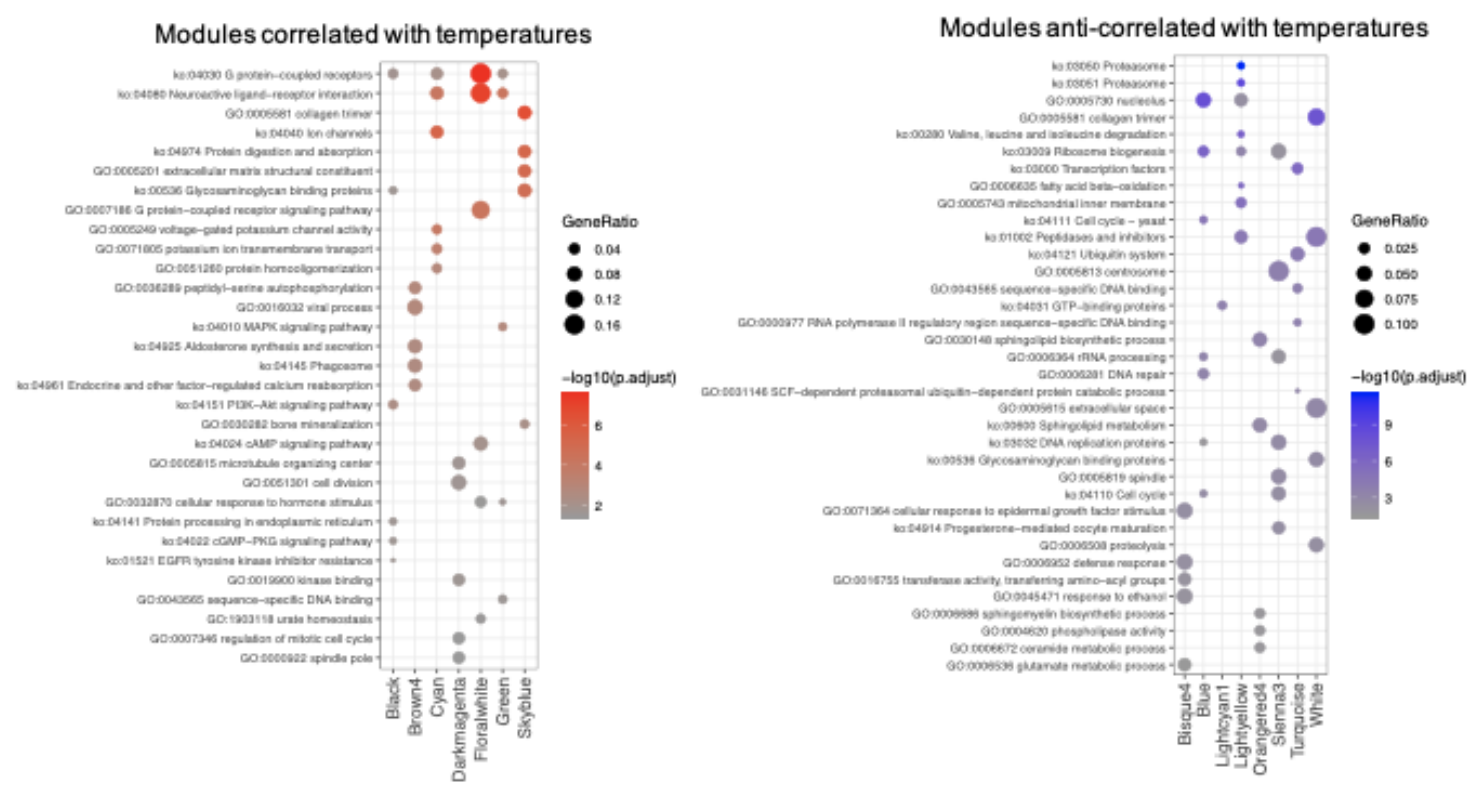

\section{Supplementary Figure 4}


bioRxiv preprint doi: https://doi.org/10.1101/2021.10.25.465660; this version posted October 26, 2021. The copyright holder for this preprint (which was not certified by peer review) is the author/funder. All rights reserved. No reuse allowed without permission.
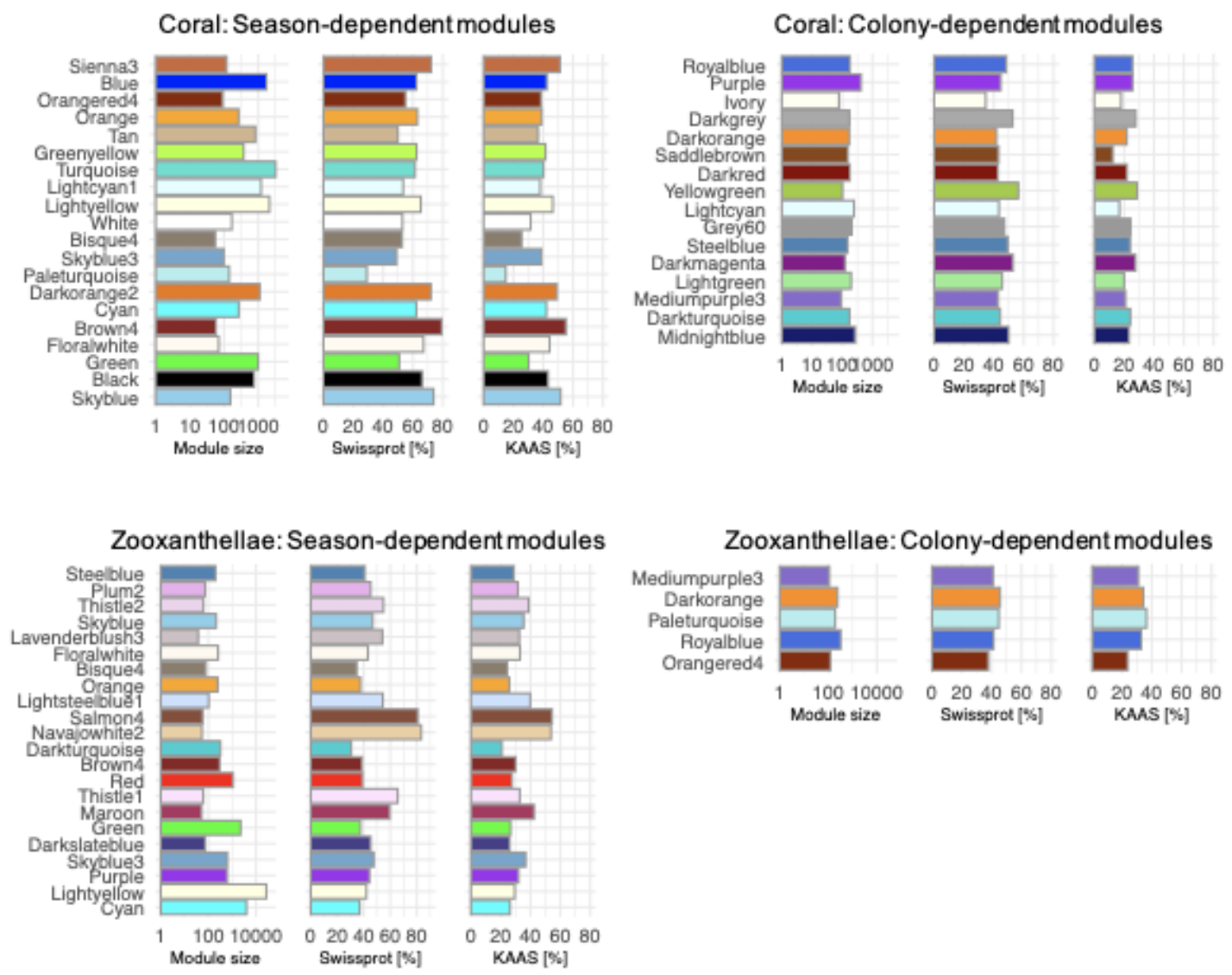

Supplementary Figure 5 
bioRxiv preprint doi: https://doi.org/10.1101/2021.10.25.465660; this version posted October 26, 2021. The copyright holder for this preprint (which was not certified by peer review) is the author/funder. All rights reserved. No reuse allowed without permission.

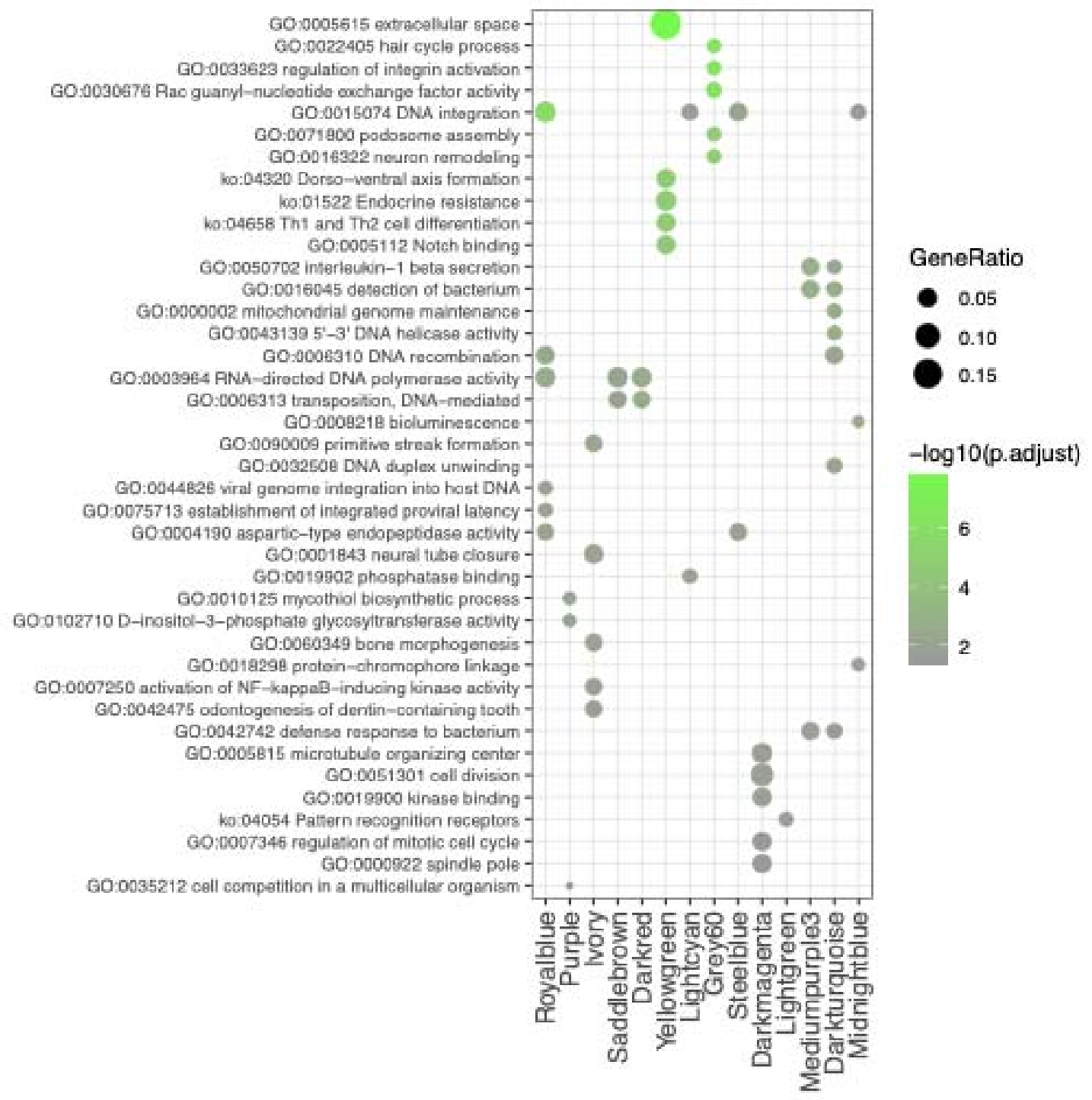

\section{Supplementary Figure 6}


bioRxiv preprint doi: https://doi.org/10.1101/2021.10.25.465660; this version posted October 26, 2021. The copyright holder for this preprint (which was not certified by peer review) is the author/funder. All rights reserved. No reuse allowed without permission.

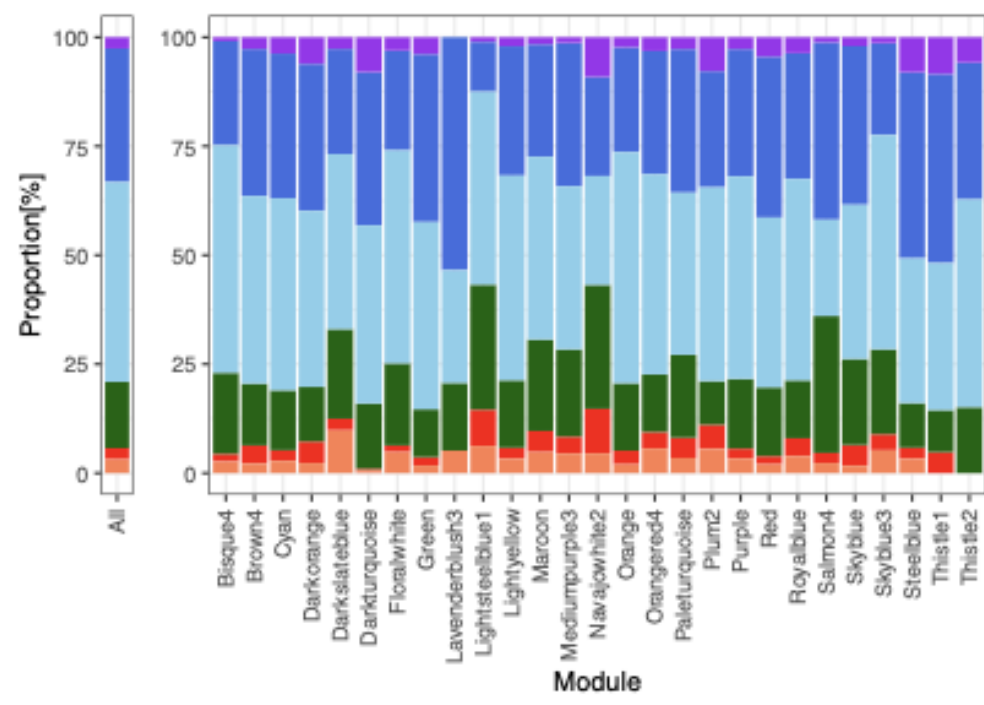

Fugacium kawagutī

Cladocopium goreaui

Cladocopium sp. clade C

Breviolum minutum

Symbiodinium microadriaticum

Symbiodinium sp. clade A3

\section{Supplementary Figure 7}


bioRxiv preprint doi: https://doi.org/10.1101/2021.10.25.465660; this version posted October 26, 2021. The copyright holder for this preprint (which was not certified by peer review) is the author/funder. All rights reserved. No reuse allowed without permission.

a

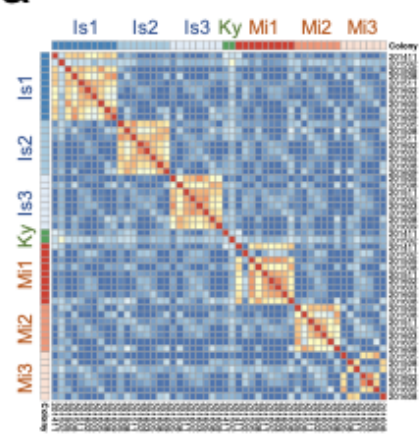

d

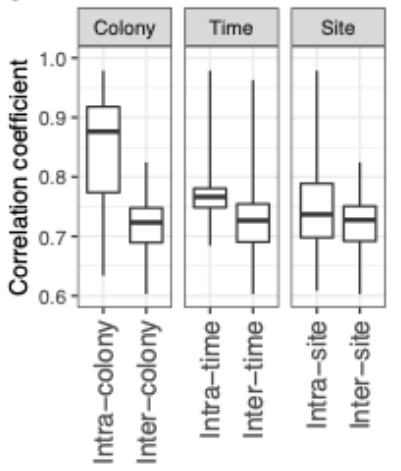

b

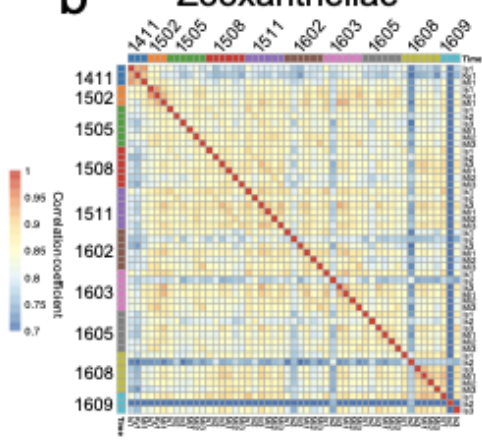

e Zooxanthellae

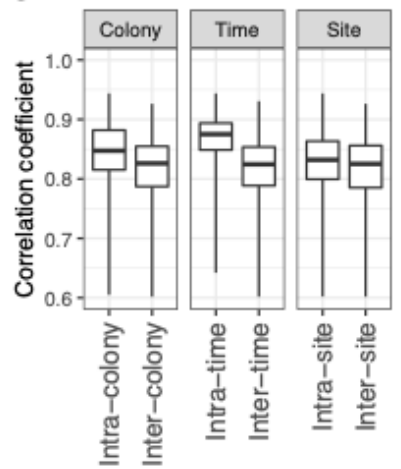

C Bacteria

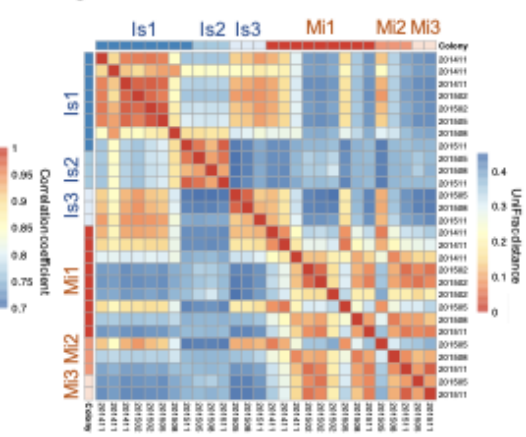

f

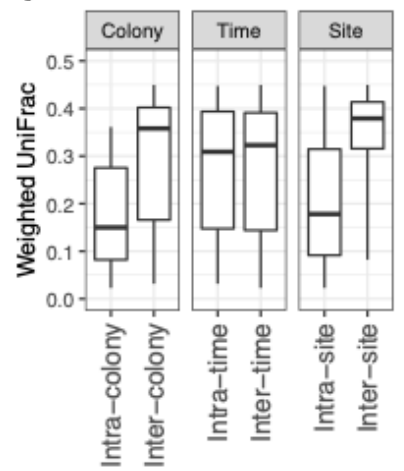

\section{Supplementary Figure 8}




\section{Tables}

Table S1 Number of collected samples

Table S2 Proportion of RNA-seq reads mapped onto A. tenuis genome

Table S3 Gene set analysis for coral gene modules with Gene Ontology

Table S4 Gene set analysis for coral gene modules with KEGG Pathway

Table S5 Gene set analysis for zooxanthellae gene modules with Gene Ontology

Table S6 Gene set analysis for zooxanthellae gene modules with KEGG Pathway

Table S7 Differentially expressed genes in corals harboring Anaplasma bacteria

Table S8 Differentially expressed genes in corals harboring Endozoicomonas bacteria

Table S9 Gene set analysis for differentially expressed genes in corals harboring Endozoicomonas

Table S10 Comparative genome analysis of Endozoicomonas species 